\title{
La turbulencia asociada con las velocidades orbitales de olas que aún no rompen
}

\author{
A.O. Hernández Olivares y F.J. Ocampo Torres \\ Departamento de Oceanografía Física, \\ Centro de Investigación Científica y de Educación Superior de Ensenada, \\ B.C. (CICESE). Carretera Ensenada-Tijuana 3918, Zona Playitas, 22860 Ensenada, B.C. \\ e-mail: ocampo@cicese.mx
}

Received 11 June 2018; accepted 11 September 2018

\begin{abstract}
Se entiende que los procesos de intercambio entre el océano y la atmósfera determinan de manera decisiva el estado del mar, del tiempo y del clima. Con el fin de entender mejor los procesos que aportan energía cinética turbulenta en las capas de fluido en ambos lados de la superficie del mar, abordamos el caso del oleaje y en particular el efecto de las olas que aún no rompen. Se realizaron mediciones de las velocidades de las partículas de fluido inducidas por la propagación de olas sin rompiente con el propósito de detectar la presencia de turbulencia y su asociación con la pendiente de las olas. Se analizaron 184 experimentos, cada uno con una duración de 3.5 minutos y olas aproximadamente monocromáticas de diferente pendiente $(0.012-0.273)$. Los experimentos se realizaron en un canal con dimensiones de $12.26 \times 0.55 \times 0.32 \mathrm{~m}$ empleando un velocímetro acústico (Vectrino Profiler, Nortek), para medir las componentes $u, v$ y $w$ de la velocidad en una columna de fluido con $3.5 \mathrm{~cm}$ de longitud en 35 celdas de $1 \mathrm{~mm}$ de ancho, aproximadamente. En cada experimento se obtuvo el perfil vertical de la velocidad entre 1.5 y $8.6 \mathrm{~cm}$ de profundidad con respecto al nivel de agua en el canal $(h=48 \pm 0.5 \mathrm{~cm})$. Se consideraron las componentes $u(t, z)$ y $w(t, z)$ en este análisis, puesto que las olas son prácticamente bidimensionales $(v(t, z)=0)$. A partir de los espectros de potencia correspondientes a las fluctuaciones turbulentas $u^{\prime}(t, z)$ y $w^{\prime}(t, z)$, se identificaron sub-rangos inerciales (turbulencia isotrópica) independientemente de la profundidad y del valor de la pendiente de las olas. Con los espectros de las fluctuaciones turbulentas de las velocidades se demuestra que el tamaño de los remolinos cuya energía es transferida a remolinos de menores escalas, es mayor al aumentar la pendiente de las olas.
\end{abstract}

Descriptores: Olas que aún no rompen; olas monocromáticas; velocímetro acústico; turbulencia; sub-rangos inerciales.

Ocean-atmosphere exchange processes are known to decisively determine the sea state, the weather and our planet's climate. With the ultimate goal of a better understanding of the processes contributing with turbulent kinetic energy into both boundary layers above and below the sea surface, we approach the ocean surface wave phenomenon, and in particular we study the non-breaking waves potential effect. Therefore, measurement of particle velocities were made in a fluid under non-breaking waves, for the purpose of detecting turbulence and its association with the wave steepness. A total of 184 experiments were analyzed, each one with a duration of 3.5 minutes and the presence of approximately monochromatic waves with varying steepness $(0.012-0.273)$. The measurements were carried out in a wave tank with dimensions of $12.26 \times 0.55 \times 0.32 \mathrm{~m}$ using an acoustic velocimeter (Vectrino Profiler, Nortek). The $u, v$ and $w$ components of velocity were measured on a $3.5 \mathrm{~cm}$ long fluid column in 35 cells of $1 \mathrm{~mm}$ in height. During the experiments, the velocity profile between 1.5 and $8.6 \mathrm{~cm}$ depth was obtained, referred to the water level in the wave tank $(h=48.6 \pm 0.5 \mathrm{~cm})$. The waves propagated in deep waters $(h / \lambda>0.5)$, where $\lambda$ is the wave length. Only $u(t, z)$ and $w(t, z)$ components were considered for the analysis since, waves are practical two-dimensional $(v(t, z)=0)$. Power spectra were calculated in as a function of frequency corresponding to $u^{\prime}(t, z)$ and $w^{\prime}(t, z)$ turbulent fluctuations, an inertial subrange (isotropic turbulence) was detected in the most of the spectra, for certain depths regardless of the wave steepness. Results from turbulent fluctuations frequency spectra show that eddy size involved in transferring energy to smaller ones, increases with the wave slope.

Keywords: Nonbreaking waves; monochromatic waves; acoustic velocimeter; turbulence; inertial subrange.

PACS: 47, 92.10hb; 94.05.Lk

\section{Introducción}

La turbulencia y la mezcla en la capa superior del océano son procesos clave en la interacción entre el océano y la atmósfera, especialmente en la transferencia de energía, calor, momento, y masa (incluyendo gases, como el $\mathrm{CO}_{2}$ ). $\mathrm{Al}$ entender de manera más completa los mecanismos que aportan turbulencia en la capa superficial del mar, incluyendo el efecto de las olas, se podrá contar con los elementos necesarios y con las cantidades apropiadas para conocer y cuantificar adecuadamente el balance de energía cinética turbulenta en ambos lados de la superficie. Así se podrá determinar de forma más precisa el intercambio entre el océano y la atmósfera, que es indispensable para predecir el estado del mar, del tiempo y el clima de nuestro planeta, con menor incertidumbre en los modelos numéricos que se utilizan. De los principales mecanismos que generan turbulencia en la capa superior del océano se pueden citar al corte vertical de las corrientes superficiales inducidas por viento y al rompimiento del oleaje $[1,2]$. El rompimiento de las olas genera turbulencia y mezcla que es confinada a profundidades del orden de las alturas de las olas e inclusive menores [3-6]. Sin embargo, también se reconoce que las olas aunque no rompan, pueden generar turbulencia en la capa superior del mar y este es el tema de investigación que se aborda en este trabajo. 
Phillips [7], propuso de manera teórica que el movimiento de las olas (aún sin romper) puede generar turbulencia asociada con la vorticidad del flujo, en donde el orden de magnitud de la vorticidad aleatoria (turbulencia) es de segundo orden en los parámetros del oleaje (como la pendiente) y por lo tanto del mismo orden que el campo promedio de la vorticidad. Posteriormente [8,9], se encuentran evidencias de generación e intensificación de la turbulencia, en respuesta al movimiento de las olas en ausencia de viento (oleaje generado en tormentas lejanas), a partir de mediciones directas en el mar. En condiciones de oleaje sin rompiente, el flujo vertical de momento horizontal debajo de la superficie excedía el esfuerzo del viento sobre la superficie del mar.

Por otra parte, considerando la teoría lineal de las olas (teoría de Airy), Babanin [10] establece un número de Reynolds para olas que aún no rompen $\left(\left[R e_{\text {wave }}\right]_{2006}=a^{2} \omega / \nu\right)$, en donde $a$ es la amplitud de las olas, y $\omega$ es su frecuencia angular, mientras que $\nu$ es la viscosidad cinemática. Determina un valor crítico $\left[R e_{\text {wave }}\right]_{2006}=3000$ que define la transición entre un flujo laminar y uno turbulento.

Además, se han desarrollado algunos estudios en el laboratorio (en canales de olas) con la finalidad de demostrar que el movimiento de las partículas en un fluido inducido por olas que aún no rompen puede generar turbulencia. Por ejemplo, Babanin y Haus [11], calcularon el espectro de potencia de la velocidad de las partículas de fluido en función del número de onda, en casos con olas monocromáticas que aún no rompen, encontrando una intermitencia en la ocurrencia de sub-rangos inerciales (Ley de $-5 / 3$ de Kolmogorov). Las velocidades fueron medidas mediante el seguimiento de partículas en el fluido a través de secuencias de imágenes en video.

Otro ejemplo de investigación en el laboratorio es el trabajo de Dai et al. [12], en donde cuantificaron la mezcla inducida por olas monocromáticas (sin rompimiento), midiendo el tiempo en que la estratificación del fluido desaparece. Utilizaron sensores de temperatura (del tipo de agujas) colocados en la mitad de un canal de $5 \mathrm{~m}$ de longitud y en diferentes profundidades, y con sus resultados demostraron que la mezcla aumenta con la amplitud y la longitud de las olas. En otro experimento, Savelyev et al. [13] midieron las velocidades de las partículas de fluido en la superficie libre $(\eta)$ bajo la presencia de olas sin romper, mediante velocimetría de marcado puntual con termografía. En este método se utilizan como trazadores del movimiento de la superficie, algunos puntos en el fluido que fueron previamente calentados con laser (Veron et al., [14]). Con sus resultados demostraron que las fluctuaciones observadas corresponden con turbulencia horizontalmente anisotrópica, debido a la formación de remolinos que se extienden en la dirección de la propagación de las olas y al aumento de su energía cinética turbulenta con el tiempo (razón de crecimiento). Finalmente, Beyá et al. [15] realizaron experimentos con olas monocromáticas de amplitud finita con valores de $\left[R e_{\text {wave }}\right]_{2006}=3000$, y mediante la inyección de tinta visualizaron la mezcla inducida por la presencia de turbulencia. Sin embargo, sus resultados no mostra- ron evidencias de mezcla turbulenta por parte del movimiento de las olas, pues identificaron que la pluma de tinta permaneció siempre visible y su grosor o espesor nominal se mantuvo invariante con respecto al tiempo.

En este trabajo se analizan resultados experimentales sobre la presencia de turbulencia generada por olas que aún no rompen en un fluido no estratificado. En particular, se busca identificar sub-rangos inerciales en los espectros de potencia de las componentes de las velocidades de las partículas del fluido, relativas al movimiento de las olas (evidencia de una turbulencia isotrópica), así como la cuantificación de los niveles de turbulencia generada en relación a la tasa de disipación de la energía cinética turbulenta. Finalmente, se intenta determinar si la frecuencia de ocurrencia de los subrangos inerciales, así como los niveles de turbulencia generada, muestran alguna relación con la pendiente de las olas.

En la Sec. 2 se describen el diseño experimental, las especificaciones del dispositivo utilizado en las mediciones, el control de la calidad de los datos adquiridos, algunas ecuaciones fundamentales consideradas y los cálculos realizados para la obtención de resultados. En la Sec. 3, se describen los resultados obtenidos con relación a los espectros de potencia de las velocidades de las partículas de fluido que tienen coherencia con la elevación de la superficie libre, y de esas velocidades que se asocian con las fluctuaciones turbulentas. Se aborda el tema de la identificación del sub-rango inercial en los espectros de potencia de las velocidades detectadas como fluctuaciones turbulentas y se presentan estimaciones de la tasa de disipación de energía cinética turbulenta en función de la pendiente de las olas. En la Sec. 4, se discuten los resultados y se presentan las conclusiones de nuestra investigación.

\section{Experimentos en el laboratorio y procesa- miento de los datos}

\subsection{Experimentos en el canal de olas}

Los experimentos del presente trabajo se llevaron a cabo en el laboratorio de olas de la Facultad de Ciencias Marinas (FCM) de la Universidad Autónoma de Baja California (UABC) en Ensenada BC, en un canal de $12.26 \mathrm{~m}$ de largo, $0.55 \mathrm{~m}$ de alto y $0.32 \mathrm{~m}$ de ancho. Se generaron olas aproximadamente monocromáticas (y prácticamente planas) en condiciones de aguas profundas mediante el movimiento de una paleta (unida al fondo del canal con una bisagra) impulsada por un motor eléctrico. Se realizaron 184 experimentos, con duración de aproximadamente 3.5 minutos cada uno, tiempo durante el cual la amplitud y el periodo de las olas generadas se mantuvieron relativamente constantes. Las características de las olas generadas difieren en cada experimento: las amplitudes (a) variaron entre 0.05 y $1.69 \mathrm{~cm}$, mientras que las frecuencias variaron entre 1.5 y $2.5 \mathrm{~Hz}$, reproduciendo una gama de pendientes en un intervalo entre 0.012 y 0.273 .

En cada experimento se realizaron mediciones entre 315 y 525 periodos de olas aproximadamente, lo que se conside- 
ra suficiente para que olas que aún no rompen generen turbulencia. Como referencia, se toma en cuenta que a partir de mediciones durante unos 15 periodos de las olas [11] se logra detectar la presencia de turbulencia isotrópica identificando sub-rangos inerciales en los espectros de potencia de las velocidades en función del número de onda. Además, en otro trabajo con mediciones durante los primeros 2.5 minutos de la propagación de las olas con valores de pendientes entre 0.08 y 0.2 , se reporta [12] un efecto notable de mezcla turbulenta.

Las velocidades de las partículas del fluido bajo la presencia de olas se midieron con un velocímetro acústico (Vectrino Profiler, Nortek) que cuenta con una frecuencia de muestreo de $100 \mathrm{~Hz}$ y una resolución espacial de $1 \mathrm{~mm}$ en la columna del fluido en donde se detecta el perfil vertical de las velocidades. Las componentes $u(t, z), v(t, z)$ y $w(t, z)$ de la velocidad de las partículas de fluido se determinaron a lo largo de esa columna de $3.5 \mathrm{~cm}$, en 35 celdas de $1 \mathrm{~mm}$ de altura cada una, en profundidades entre 1.5 y $8.6 \mathrm{~cm}$ con respecto al nivel medio del agua. La primer celda se obtiene a $4 \mathrm{~cm}$ sobre el transductor principal del sensor. El sensor cuenta también con un detector del fondo con una tasa de muestreo de $10 \mathrm{~Hz}$, que en este caso se utilizó como detector de la superficie del agua.

El nivel del agua en el canal fue $h=48.6 \pm 0.5 \mathrm{~cm}$, con lo que se determina [16] que en los experimentos realizados las olas se propagaron en aguas profundas i.e. $h / \lambda>0.5$. Considerando $h$ y la frecuencia del pico $\left(f_{p}\right)$ en los espectros de potencia de la componente $u(t, z)$ de las velocidades (esa frecuencia coincide con la del movimiento oscilatorio de la paleta), se determinó el número de onda ( $\left.k_{\text {wave }}\right)$ de las olas mediante la relación de dispersión.

Con relación al funcionamiento del sensor utilizado, se consideró conveniente agregar grano de café (finamente molido) al fluido, previamente a la realización de cada experimento, con lo que se logró obtener una mayor amplitud en la señal acústica reflejada y valores apropiados en la razón de señal a ruido $(\geq 35 \mathrm{~dB})$.

Para reducir la reflexión de las olas al final de su propagación, en el extremo del canal se colocó una plataforma de madera perforada (simulando una playa), con una pendiente de $8^{\circ}$ aproximadamente y cubierta con pasto sintético. El fondo y las paredes del canal son de vidrio, por lo que pueden considerarse como superficies lisas. El transductor del sensor utilizado se colocó en el centro del canal, aproximadamente a $6 \mathrm{~m}$ de la paleta y a $0.15 \mathrm{~m}$ de las paredes. La distancia a la paleta se considera suficiente para que las olas con pendientes menores que 0.3 se propaguen sin modificación visible de su perfil, mientras que la distancia a las paredes se considera suficiente como para evitar cualquier efecto en las mediciones de la velocidad por causa de la capa límite.

El sensor se instaló de modo que el transductor perfilara hacia arriba y con el detector del fondo se logró determinar la elevación de la superficie libre, $\eta(t)$. Las series temporales $\eta(t)$, se interpolaron para obtener correspondencia con las series $u(t, z), v(t, z)$ y $w(t, z)$. Además, se calcularon los es- pectros de potencia de $\eta(t)\left(S_{\eta \eta}(f)\right)$, y la amplitud correspondiente como $A(f)=\sqrt{2 S_{\eta \eta}(f) \Delta f}$ [17,18], en donde $\Delta f$ es el ancho de la banda de frecuencias que describe la resolución en el espectro. En este trabajo, la altura de las olas que se utiliza es $H=2$ máx $(A(f))$, donde máx es la función de valor máximo.

En cada experimento, el sensor de las velocidades se encendió aproximadamente 10 segundos después de iniciar la generación de las olas, tiempo suficiente para que el movimiento de la paleta se considere regular. Una vez finalizado el tiempo de medición, el generador de oleaje se apagó y se esperó que las últimas olas generadas se disiparan por completo antes de proceder con el siguiente experimento (aproximadamente $10 \mathrm{~min}$ ).

\subsection{Control de calidad de los datos adquiridos}

Además de las mediciones de las componentes $u(t, z), v(t, z)$ y $w(t, z)$ de las velocidades de las partículas de fluido, el sensor proporciona coeficientes de correlación del haz acústico (una matriz por cada haz acústico). Estos coeficientes de correlación del haz acústico son una medida de la similitud entre dos pulsos acústicos emitidos sucesivamente y representan también una medida de la calidad en las mediciones de velocidad [19].

En este trabajo se consideran como ruido, los datos de velocidad cuyos valores de correlación del haz acústico son menores que $75 \%$ en cualquiera de esas cuatro matrices de correlación. Los valores identificados como ruido se sustituyen con NaNs (Not a number), al igual que los valores de las velocidades fuera del intervalo $[-3 \sigma+\mu, \mu+3 \sigma]$, donde $\mu$ corresponde a la media temporal de la serie de tiempo y $\sigma$ a su desviación estándar.

Las series de tiempo cuyo total de NaNs sea mayor que $20 \%$ del total de sus datos originales deben descartarse. Sin embargo, en nuestros experimentos, todas las series de tiempo fueron consideradas para su análisis puesto que el total de NaNs fué siempre menor que $20 \%$. Las series de datos de las velocidades se interpolaron en el tiempo empleando el método polinómico de Hermite para sustituir los NaNs por valores de velocidad.

Con los ángulos de Euler obtenidos a partir de las series de $u(t, z), v(t, z)$ y $w(t, z)$, se construyeron las matrices de rotación de coordenadas con las cuales se corrigió la orientación e inclinación del sensor.

Con el propósito de determinar la intensidad de la posible reflexión de las olas en el canal durante la realización de nuestros experimentos, se obtuvieron espectros direccionales $D(f, \theta)$, en función de la frecuencia $f$ y la dirección de propagación $\theta$, utilizando las series de tiempo de $u(t, z)$, $v(t, z)$ y $w(t, z)$ y el conjunto de rutinas computacionales denominado Directional Wave Spectra (DIWASP) para Matlab versión 1.4, para aplicar el método extendido de máxima entropía [20]. En cada profundidad z en donde se midieron las velocidades, se estimó un espectro direccional $D(f, \theta)$. Me- 
diante $D(f, \theta)$, se determinó la energía incidente de las olas $E_{I}$ y la reflejada $E_{R}[17,18]$ de la forma:

$$
\begin{aligned}
E_{I} & =\int_{0}^{f \text { máx }} D\left(f, \theta_{I}\right) d f \\
E_{R} & =\int_{0}^{f \text { máx }} D\left(f, \theta_{R}\right) d f
\end{aligned}
$$

donde $\theta_{I}$ corresponde al ángulo que define la dirección incidente de las olas i.e. la dirección correspondiente a la frecuencia del pico en $D(f, \theta), \theta_{R}$ es el ángulo que define la dirección directamente opuesta a $\theta_{I}$ y $f_{\text {máx }}$ es la frecuencia máxima resuelta $(6 \mathrm{~Hz})$ en los $D(f, \theta)$, la cual es previamente definida por el usuario en las rutinas DIWASP. Una $f_{\text {máx }}=6$ $\mathrm{Hz}$ se considera suficiente para determinar $E_{I}$ y $E_{R}$ de las olas durante los experimentos, con lo que se calculó el coeficiente de reflexión $K_{R}=\left(E_{R} / E_{I}\right)^{1 / 2}[17,18,21]$, correspondiente a cada $z$.

Los 184 experimentos fueron considerados para el análisis de turbulencia bajo la premisa de que $K_{R}(z)<0.15$ no corresponde a una reflexión significativa de las olas. En nuestros experimentos, los valores de $K_{R}(z)$ presentaron un promedio $\mu=0.034 \pm 0.018$.

En este trabajo, únicamente se analizan las componentes $u(t, z)$ y $w(t, z)$ de las velocidades de las partículas del fluido, pues se considera que el campo de las velocidades orbitales de las olas es bi-dimensional i.e. $v(t, z)=0$. Las series temporales de $v(t, z)$ no mostraron un comportamiento característico de una velocidad orbital y la raíz cuadrática media de $v(t, z)\left(v(z)_{r m s}\right)$ presentó valores entre 1 y 3 órdenes de magnitud menor que $u(z)_{r m s}$ y $w(z)_{r m s}$. Además, el promedio temporal de $v(t, z)$ es prácticamente igual a cero $\overline{(v(z)}=0)$.

\section{Aspectos teóricos fundamentales y el cálcu- lo de características de la turbulencia}

\subsection{Espectros de las velocidades en fluidos turbulentos}

El espectro de potencia de la velocidad en un flujo turbulento homogéneo e isotrópico con números de Reynolds grandes ( $\operatorname{Re}>3000)$, presenta un intervalo característico denominado sub-rango inercial o de Kolmogorov [22]. La densidad de energía espectral en el sub-rango inercial, se puede expresar como:

$$
E(k)=\alpha \varepsilon^{2 / 3} k^{-5 / 3}
$$

donde $\varepsilon$ es la tasa de disipación de la energía cinética turbulenta, $k$ es el módulo del vector número de onda y $\alpha$ la constante de Kolmogorov cuyo valor se ha determinado entre 1.4 y 2.2 [23]. En el sub-rango inercial, la energía contenida en los remolinos turbulentos de macro-escala se transfiere a remolinos de menores escalas en un proceso denominado cascada de energía, obedeciendo a una ley de transferencia de energía conocida como la ley de $k^{-5 / 3}$ o Ley de Kolmogorov.

La ley de $k^{-5 / 3}$ se cumple en espectros unidimensionales correspondientes a una de las componentes de la velocidad e.g. $E_{u u}\left(k_{1}\right)=\left(\alpha_{1} / 2\right) \varepsilon^{2 / 3} k_{1}^{-5 / 3}$, donde $E_{u u}\left(k_{1}\right)$ es el espectro de la componente horizontal de la velocidad en dirección paralela al flujo medio, $k_{1}$ la componente horizontal del vector número de onda $\mathbf{k}=\left(k_{1}, k_{2}, k_{3}\right)$ y $\alpha_{1} \approx 0.4$ una constante denominada constante de Heisenberg [24].

La turbulencia puede considerarse como congelada, si la intensidad media de las fluctuaciones turbulentas es al menos un orden de magnitud menor que la intensidad de la velocidad del flujo medio i.e. $u_{\mathrm{rms}}^{\prime}, w_{\mathrm{rms}}^{\prime} \ll U$, donde $u_{\mathrm{rms}}^{\prime} \mathrm{y} w_{\mathrm{rms}}^{\prime}$ son respectivamente la raíz cuadrática media (intensidad) de las fluctuaciones turbulentas de las componentes $u$ y $w$ de la velocidad y $U$ es la velocidad del flujo medio. En este caso, las fluctuaciones turbulentas de la velocidad se transportan con el flujo medio a una velocidad $U$, i.e. Hipótesis de Taylor [25].

Bajo las condiciones de turbulencia congelada, los espectros de potencia de las velocidades en función del número de onda pueden ser expresados en función de la frecuencia mediante la hipótesis de Taylor [26], tal que $\omega=2 \pi f=k_{1} U$ e.g. $E_{u u}(f)=(\pi / U) E_{u u}\left(k_{1}\right)$, donde $E_{u u}(f)$ es el espectro de la componente horizontal de la velocidad en función de la frecuencia $f$ y $\omega$ la frecuencia angular, así mismo la ley de $k^{-5 / 3}$ puede ser expresada en función de la frecuencia como $f^{-5 / 3}$.

Benilov y Filyushkin [27] expresan el espectro de potencia de las fluctuaciones turbulentas de las velocidades de las partículas de un fluido bajo la presencia de olas como:

$$
S_{u^{\prime} u^{\prime}}(f)=S_{u u}(f)-S_{\tilde{u} \tilde{u}}(f)
$$

donde $S_{u u}(f)$ corresponde al espectro de potencia de la componente $u$ de las velocidades instantáneas, $S_{\tilde{u} \tilde{u}}(f)$ al espectro de potencia de la componente $\tilde{u}$ de las velocidades orbitales asociadas con el oleaje y $S_{u^{\prime} u^{\prime}}(f)$ al espectro de potencia de la componente $u^{\prime}$ de las velocidades turbulentas.

Thornton [28] define el espectro de las velocidades orbitales asociadas con el oleaje como:

$$
S_{\tilde{u} \tilde{u}}(f)=S_{u u}(f) \times C_{u \eta}(f)^{2}
$$

donde $C_{u \eta}(f)^{2}$ corresponde a la coherencia espectral entre la componente $u(t)$ de la velocidad instantánea y análogamente, el espectro de potencia de la componente $\tilde{w}$ de las velocidades orbitales asociadas con el oleaje se expresa como $S_{\tilde{w} \tilde{w}}(f)=S_{w w}(f) \times C_{w \eta}(f)^{2}$.

A partir de las series de tiempo de las componentes $u(t, z)$ y $w(t, z)$ de las velocidades medidas en los experimentos realizados en el canal de olas, se calcularon los espectros de potencia $S_{u u}(f)$ y $S_{w w}(f)$ en cada profundidad $z$ (35 espectros $S_{u u}(f)$ y $S_{w w}(f)$ en cada experimento) con $2 K=50$ grados de libertad, donde $K$ es el número de segmentos o particiones de las series de tiempo. Se calcularon 
también las respectivas coherencias espectrales con la elevación de la superficie libre $\eta(t),\left(C_{u \eta}(f)^{2}\right)$ y $\left(C_{w \eta}(f)^{2}\right)$ obtenida a partir de la señal del detector del fondo del sensor utilizado. Posteriormente, considerando las expresiones (4) y de manera análoga (5), se obtuvieron los espectros de potencia $S_{u^{\prime} u^{\prime}}(f)$ y $S_{w^{\prime} w^{\prime}}(f)$.

Se realizó un análisis de la forma de cada espectro $S_{u^{\prime} u^{\prime}}(f, z)$ y $S_{w^{\prime} w^{\prime}}(f, z)$ para identificar la región en el dominio de frecuencias, en donde se presentó el sub-rango inercial tal que $S_{u^{\prime} u^{\prime}}(f) \sim f^{-5 / 3}$ y $S_{w^{\prime} w^{\prime}}(f) \sim f^{-5 / 3}$. Se obtuvo un ajuste con el método de mínimos cuadrados del valor de $S$ (en escala logarítmica) y se determinó si la pendiente correspondiente es $m=-5 / 3 \pm 0.04$, con lo que se satisface la ley de $f^{-5 / 3}$ (en este caso, el valor de tolerancia es \pm 0.04 , con lo que se obtiene un $95 \%$ de confiabilidad). La identificación de la pendiente $m(-5 / 3 \pm 0.04)$ se realizó con los valores de la densidad de energía espectral, en un rango de frecuencias $\geq 15 \mathrm{~Hz}$ (ampliando el rango hasta una frecuencia máxima en donde la correlación entre $S$ y $f^{-5 / 3}$ se reduce hasta valores menores que 0.8 ).

Lumley y Terray [29], demostraron que la raíz cuadrática media de las velocidades de las partículas en un fluido bajo condiciones de olas unidireccionales en aguas profundas, resulta adecuada para utilizar la relación de Taylor i.e. $\omega=k_{1} U_{\mu}$, donde $U_{\mu}$ es la raíz cuadrática media de la velocidad instantánea expresada como:

$$
U_{\mu}(z)=\left(\frac{1}{N}\left(\sum_{i=1}^{N} u\left(t_{i}, z\right)^{2}+w\left(t_{i}, z\right)^{2}\right)\right)^{1 / 2}
$$

La intensidad de cada componente de las fluctuaciones turbulentas $\left(u_{r m s}^{\prime}\right.$ y $\left.w_{r m s}^{\prime}\right)$ se estimó a partir de $S_{u^{\prime} u^{\prime}}(f)$ y $S_{w^{\prime} w^{\prime}}(f)$ mediante la relación de Parseval. Los resultados de $U_{\mu}(z)$ presentaron valores (entre 10 y 60 veces) mayores que $u_{r m s}^{\prime}$ y $w_{r m s}^{\prime}$, con lo que se sugiere considerar la existencia de turbulencia congelada en nuestros experimentos.

En atención a la turbulencia congelada y mediante la relación $2 \pi f=k_{1} U_{\mu}$, se realizó la conversión de $f$ a $k_{1}$ en los sub-rangos inerciales identificados en $\operatorname{los} S_{u^{\prime} u^{\prime}}(f)$ y $S_{w^{\prime} w^{\prime}}(f)$. Los espectros de potencia $S_{u^{\prime} u^{\prime}}(f)$ y $S_{w^{\prime} w^{\prime}}(f)$ también se transformaron al dominio del número de onda, para estimar $S_{u^{\prime} u^{\prime}}\left(k_{1}\right)$ y $S_{w^{\prime} w^{\prime}}\left(k_{1}\right)$, respectivamente y posteriormente utilizarse en el análisis.

\subsection{Tasa de disipación de la energía cinética turbulenta}

La tasa de disipación de energía cinética turbulenta $\varepsilon$, incluida en la expresión del sub-rango inercial del espectro de potencia de la velocidad (ver Ec. 3), es una característica de la cantidad de turbulencia en un fluido y de la cantidad de mezcla generada por esa turbulencia, especialmente al tratar un caso estacionario cuando existe un equilibrio entre la generación y la disipación de esa energía cinética turbulenta. $\mathrm{La}$ tasa de disipación $\varepsilon$, asociada a cada componente de las fluctuaciones turbulentas de la velocidad, se puede estimar a partir de las expresiones del sub-rango inercial de los espectros de potencia unidimensionales en función de la frecuencia (en unidades de densidad de energía espectral) correspondientes a cada una de las componentes de la velocidad turbulenta $u^{\prime}$ y $w^{\prime}$ mediante:

$$
\varepsilon_{u^{\prime}}(z)=\mathcal{C} 2 \pi U_{\mu}(z)^{-1} f^{5 / 2} S_{u^{\prime} u^{\prime}}(f, z)^{3 / 2}
$$

y

$$
\varepsilon_{w^{\prime}}(z)=\mathcal{C} 2 \pi U_{\mu}(z)^{-1} f^{5 / 2} S_{w^{\prime} w^{\prime}}(f, z)^{3 / 2}
$$

en donde $\mathcal{C}$ corresponde a una constante del orden de la unidad, y en este trabajo se considera $\operatorname{como} \mathcal{C}=1$. Para una
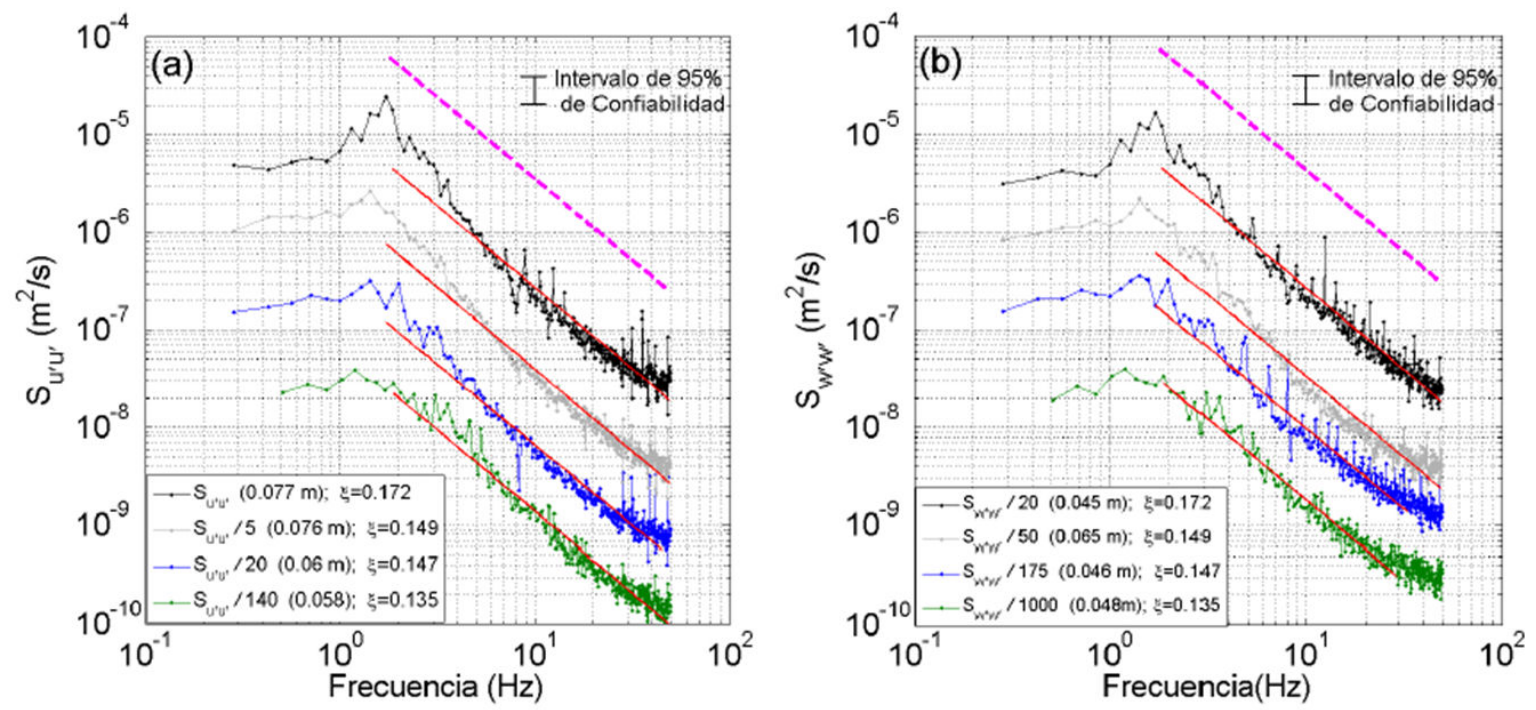

FIGURE 1. Ejemplo de espectros de potencia en donde se muestran sub-rangos inerciales en a) $S_{u^{\prime} u^{\prime}}(f, z)$ y b) $S_{w^{\prime} w^{\prime}}(f, z)$ de 4 experimentos diferentes. La línea magenta segmentada corresponde a la ley de $f^{-5 / 3}$ de Kolmogorov y la línea roja a un intervalo de frecuencia con $m=-5 / 3 \pm 0.04$. Cada color en los espectros de potencia especifica un experimento en particular para una profundidad y $\xi$ determinada. 
mayor certeza en la estimación de $\varepsilon$, se realizó un promedio en frecuencia en (7) y (8).

De acuerdo con la teoría de Kolmogorov, la ley de $k_{1}^{-5 / 3}$ es apreciable dentro del intervalo $2 \pi / L \ll k_{1}^{-5 / 3} \ll 2 \pi / n_{k}$, donde $L$ representa la dimensión longitudinal de la macroescala de la turbulencia (escala integral), y $2 \pi / L$ corresponde con el menor número de onda en ese intervalo y el que contiene la mayor energía en el espectro. En el otro extremo de ese intervalo, la micro-escala de la turbulencia (micro-escala de Kolmogorov) se define como $n_{k}=\left(\nu^{3} / \varepsilon\right)^{1 / 4}$, donde $\nu$ es la viscosidad cinemática del agua y puede estimarse a partir de los valores de $\varepsilon$ que se obtienen de los sub-rangos iner- ciales identificados en $S_{u^{\prime} u^{\prime}}(f)$ y $S_{w^{\prime} w^{\prime}}(f)$. La micro-escala de Kolmogorov representa el tamaño de los remolinos más pequeños en el campo turbulento, es en dicha escala que los efectos de la disipación viscosa son de los más intensos.

\section{Resultados}

En las Figs. 1a y 1b se presentan ejemplos de los espectros $S_{u^{\prime} u^{\prime}}(f)$ y $S_{w^{\prime} w^{\prime}}(f)$, respectivamente, obtenidos a partir de 4 experimentos con pendiente de las olas entre 0.135 y 0.172 . El conjunto de espectros de un color corresponde a un experimento en particular y a una determinada profundidad.
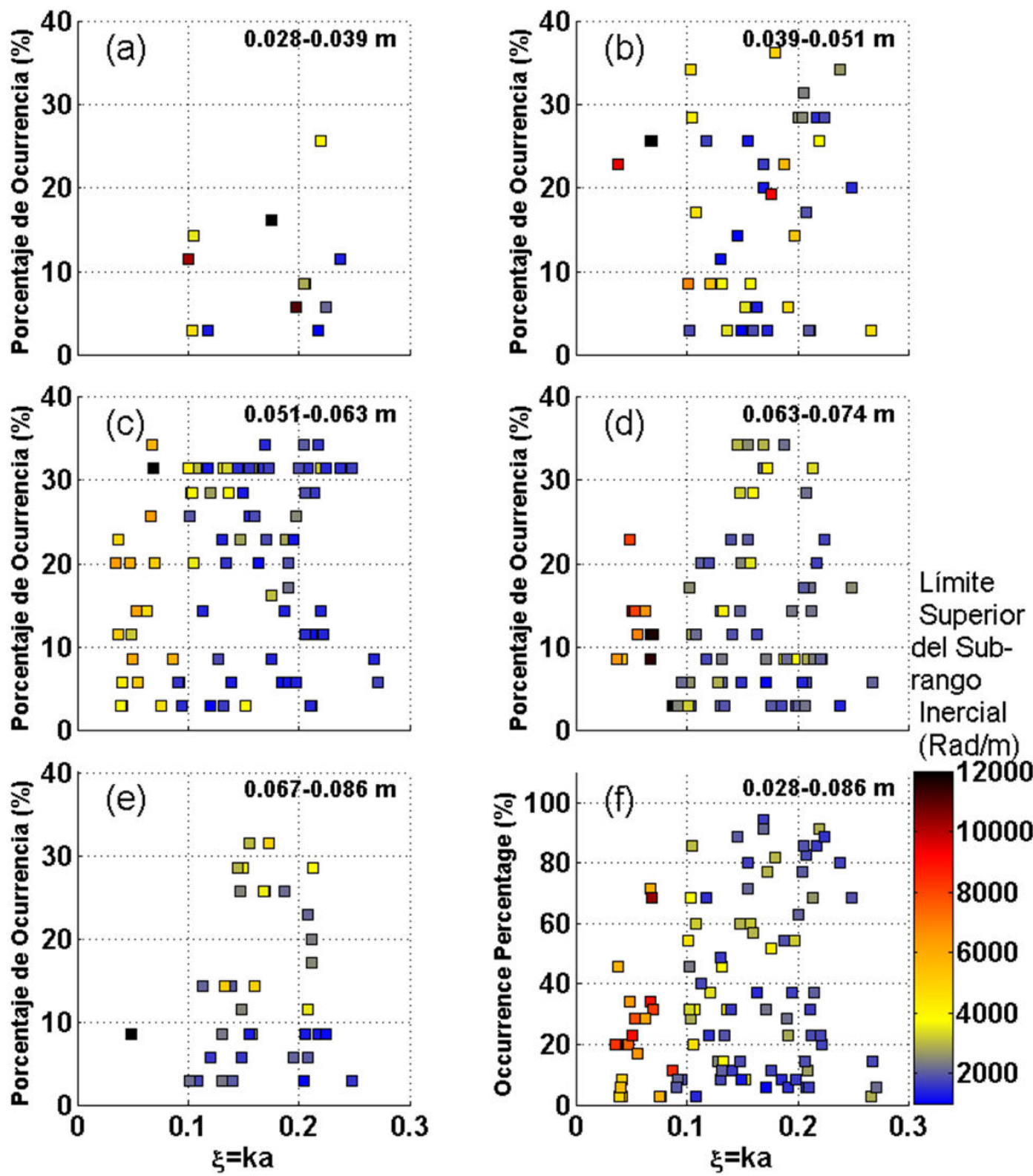

FIGURE 2. Porcentaje de ocurrencia de sub-rangos inerciales en los espectros $S_{u^{\prime} u^{\prime}}\left(k_{1}, z\right)$ en función de $\xi$. Los incisos a, b, c, d y e corresponden a diferentes intervalos de profundidad dentro del rango de perfilamiento $0.028-0.086 \mathrm{~m}$ (inciso f). El patrón de colores especifica el número de onda límite superior promedio (en $\mathrm{rad} / \mathrm{m}$ ) de los sub-rangos inerciales identificados. 

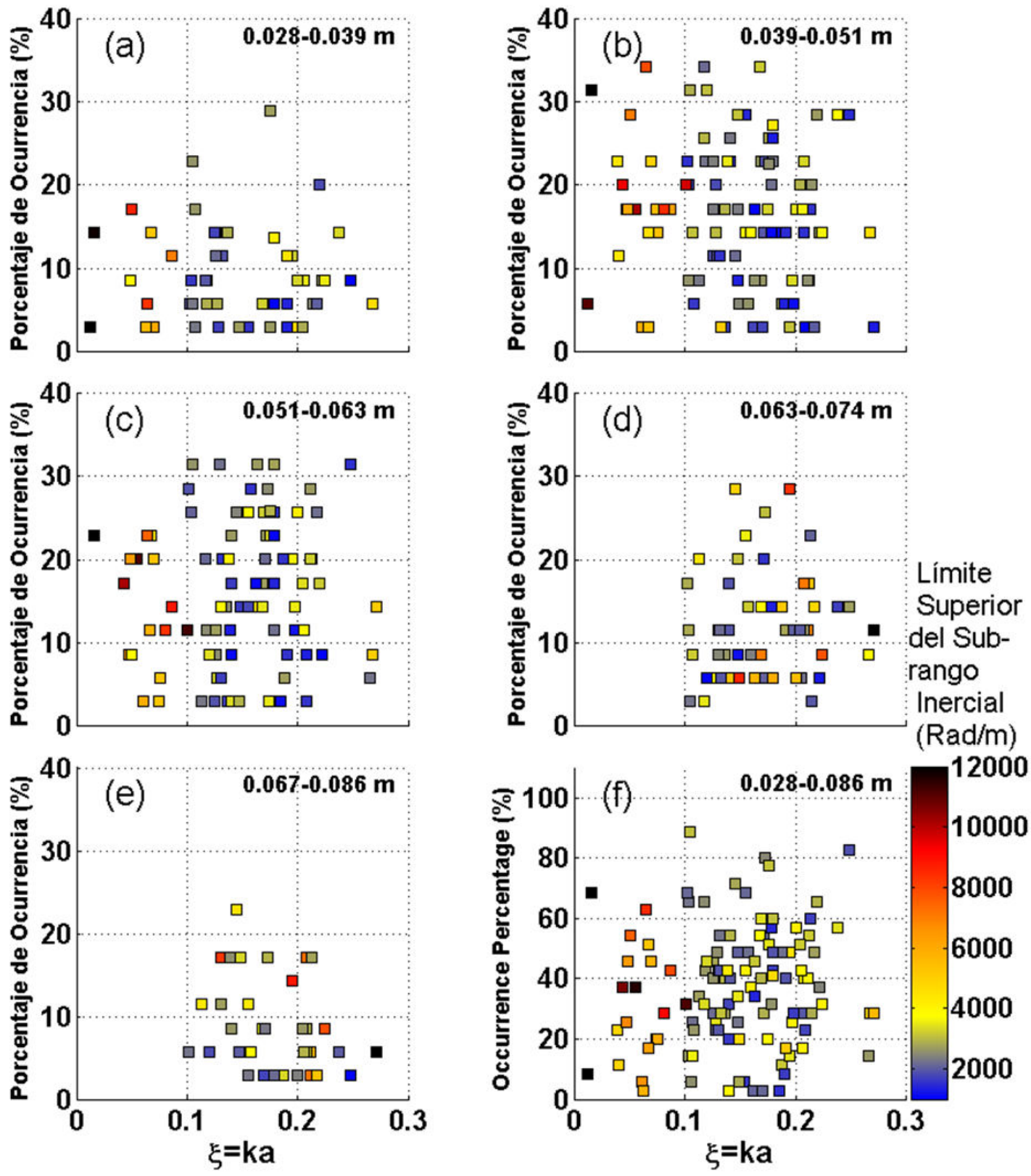

FIGURE 3. Porcentaje de ocurrencia de sub-rangos inerciales en los espectros $S_{w^{\prime} w^{\prime}}\left(k_{1}\right)$ en función de $\xi$. Los incisos a, b, c, d y e corresponden a diferentes intervalos de profundidad dentro del rango de perfilamiento $0.028-0.086 \mathrm{~m}$ (inciso f). El patrón de colores especifica el número de onda límite superior promedio (en $\mathrm{rad} / \mathrm{m}$ ) de los sub-rangos inerciales identificados.

En color negro los resultados del experimento $\mathrm{n}^{\circ} 101$, con $\xi=0.172$; en color gris, del experimento $\mathrm{n}^{\circ} 91$, con $\xi=$ 0.149 ; en azul, del experimento $\mathrm{n}^{\circ} 105$, con $\xi=0.147$ y en verde, los resultados del experimento $\mathrm{n}^{\circ} 40, \operatorname{con} \xi=0.135$.

La línea segmentada magenta indica $S \sim f^{-5 / 3}$ que corresponde a la ley de Kolmogorov. La línea roja en cada conjunto de espectros representa el ajuste de la energía espectral de un caso en particular como ejemplo, en donde se identifica el sub-rango inercial con $m=-5 / 3 \pm 0.04$.

La Fig. 2, presenta el porcentaje del total (184 experimentos) de los espectros $S_{u^{\prime} u^{\prime}}\left(k_{1}, z\right)$ (una vez transformados al dominio de $k_{1}$ bajo la consideración de una turbulencia congelada), en donde se identificó la presencia del sub-rango inercial. Los resultados se describen también en función de la pendiente de las olas $(\xi)$ y de los intervalos verticales que corresponden con profundidad: a) entre 0.028 y $0.039 \mathrm{~m}, \mathrm{~b}$ ), entre 0.039 y $0.051 \mathrm{~m}, \mathrm{c}$ ) entre 0.051 y $0.063 \mathrm{~m}, \mathrm{~d}$ ) entre 0.063 y $0.074 \mathrm{~m}$ y e) entre 0.063 y $0.086 \mathrm{~m}$, y en el perfil completo que corresponde con profundidades f) entre 0.028 y $0.086 \mathrm{~m}$. Un valor de $100 \%$ indica que en ese experimento en particular se identificó la presencia de sub-rango inercial en todos y cada uno de los espectros en todas las profundidades en las que se realizaron las mediciones. El patrón de colores especifica el promedio (del total de los experimentos) 
del número de onda correspondiente al límite superior del sub-rango inercial (en el dominio de $k_{1}$ ) identificado en cada intervalo y rango de profundidad indicados. Se identificó la presencia del sub-rango inercial en los espectros $S_{u^{\prime} u^{\prime}}\left(k_{1}\right)$ con una cierta variabilidad en el número de onda límite superior, dependiendo de los diferentes intervalos de profundidad dentro de la gama completa de valores de $\xi(0.012-0.273)$. El mayor número de experimentos cuyos $S_{u^{\prime} u^{\prime}}\left(k_{1}\right)$ presentaron sub-rangos inerciales, se observó en profundidades entre 0.051 y $0.074 \mathrm{~m}$. Particularmente, el $46 \%$ de los experimentos presentó sub-rangos inerciales en $S_{u^{\prime} u^{\prime}}\left(k_{1}\right)$ en profundidades entre 0.051 y $0.063 \mathrm{~m}$ (Fig. 2c) y entre 0.067 y $0.074 \mathrm{~m}$ (Fig. 2d). En profundidades entre 0.028 y $0.039 \mathrm{~m}$ (Fig. 2a) y entre 0.067 y $0.086 \mathrm{~m}$ (Fig. 2e), se presentó la menor cantidad de experimentos con ocurrencia de sub-rangos inerciales en los $S_{u^{\prime} u^{\prime}}\left(k_{1}\right)$ (7\% y $19 \%$ respectivamente), sugiriendo una relativa ausencia de turbulencia. El $53 \%$ de los experimentos presentó sub-rangos inerciales en los $S_{u^{\prime} u^{\prime}}\left(k_{1}\right)$ en profundidades entre 0.028 y $0.086 \mathrm{~m}$ (Fig. 2f).

En las Figs. 3a, b, c, d y e, se presenta el porcentaje de ocurrencia de sub-rangos inerciales en los espectros de las componentes verticales de las velocidades turbulentas $S_{w^{\prime} w^{\prime}}\left(k_{1}\right)$, en función de $\xi$, y se indica también el número de onda límite superior de ese sub-rango. Se muestran los resultados obtenidos dentro de los intervalos de profundidad indicados, en el rango $0.028-0.086 \mathrm{~m}$ de la columna en donde se realizaron las mediciones. En profundidades entre 0.039 y $0.063 \mathrm{~m}$ se encontró la mayor cantidad de experimentos cuyos $S_{w^{\prime} w^{\prime}}\left(k_{1}\right)$ presentaron sub-rangos inerciales. Particularmente, $55 \%$ de los experimentos presentaron subrangos inerciales en profundidades entre 0.039 y $0.051 \mathrm{~m}$ (Fig. 3b) y el $47 \%$ de los experimentos entre 0.051 y 0.063 $\mathrm{m}$ (Fig. 3c). En los intervalos de profundidad entre 0.028 y $0.039 \mathrm{~m}$ (Fig. 3a) y entre 0.067 y $0.086 \mathrm{~m}$ (Fig. 3e) se presentó la menor cantidad de experimentos con ocurrencia de sub-rango inercial en $S_{w^{\prime} w^{\prime}}\left(k_{1}\right)$ (26\% y $18 \%$ respectivamente) lo que sugiere menor presencia de turbulencia. Dentro del rango completo de profundidades $0.028-0.086 \mathrm{~m}$ (Fig. 3f), el $61 \%$ de los experimentos presentó sub-rangos inerciales en $S_{w^{\prime} w^{\prime}}\left(k_{1}\right)$.

\section{Discusión y comentarios finales}

En los espectros de potencia $S_{u^{\prime} u^{\prime}}(f)$ y $S_{w^{\prime} w^{\prime}}(f)$ estimados a partir de las mediciones en el laboratorio, se identificaron sub-rangos inerciales en $53 \%$ y $61 \%$ del total de experimentos respectivamente. En ninguno de los intervalos de profundidad en el rango de las mediciones se encontró relación entre el ancho en frecuencia de los sub-rangos inerciales identificados y los valores de $\xi$. Sin embargo, en los sub-rangos inerciales en función de $k_{1}$, el ancho que se observa corresponde a números de onda que disminuyen al aumentar los valores de $\xi$ (ver Figs. 2c-f y 3a-c, f). Esto sugiere que el proceso de cascada de energía en $S_{u^{\prime} u^{\prime}}\left(k_{1}\right)$ y $S_{w^{\prime} w^{\prime}}\left(k_{1}\right)$, ocurre en remolinos turbulentos de mayor tamaño en las olas con mayor $\xi$. Además, se identificó que la variabilidad del ancho del sub-rango inercial en el dominio de $k_{1}$ en función de $\xi$ puede ser predominante en ciertos intervalos de profundidad (ver Figs. 2a, b y $3 \mathrm{~d}$, e), con lo que se enmascara de manera intermitente la presencia de la cascada de energía ya mencionada. En términos generales, el proceso de cascada de energía se extiende hasta remolinos con menores (mayores) escalas al disminuir (aumentar) $\xi$.

El número de onda límite superior del sub-rango inercial identificado en $S_{u^{\prime} u^{\prime}}\left(k_{1}\right)$ y $S_{w^{\prime} w^{\prime}}\left(k_{1}\right)$ es menor que $2 \pi / n_{k}$, en particular en $70 \%$ de $S_{u^{\prime} u^{\prime}}\left(k_{1}\right)$ y $66 \%$ de $S_{w^{\prime} w^{\prime}}\left(k_{1}\right)$ donde se identificó el sub-rango inercial, el valor de su límite superior resultó ser de un orden de magnitud menor que $2 \pi / n_{k}$ cumpliendo con la hipótesis de Kolmogorov, los valores de $k_{1} \geq 2 \pi / n_{k}$ definen el dominio en $S_{u^{\prime} u^{\prime}}\left(k_{1}\right)$ y $S_{w^{\prime} w^{\prime}}\left(k_{1}\right)$ donde los efectos de la disipación viscosa son dominantes (rango de disipación).

La presencia del sub-rango inercial en los espectros analizados es independiente de la pendiente de las olas, sin embargo en $S_{u^{\prime} u^{\prime}}\left(k_{1}\right)$ se identificaron sub-rangos inerciales con una mayor frecuencia de ocurrencia en mediciones con profundidades entre 0.051 y $0.074 \mathrm{~m}$, mientras que en $S_{w^{\prime} w^{\prime}}\left(k_{1}\right)$ la mayor ocurrencia se identificó en las mediciones con profundidades entre 0.039 y $0.063 \mathrm{~m}$. Babanin y Hauss [11], presentaron resultados del sub-rango inercial identificado en espectros de potencia de la componente $u$ de la velocidad en función del número de onda. El sub-rango inercial fué identificado en tres ocasiones a partir de las mediciones de cada experimento con una duración de aproximadamente 15 periodos de las olas (10 segundos). La pendiente de las olas en sus experimentos fue de $0.13 \leq \xi \leq 0.25$. En los resultados del presente trabajo, el análisis llevado a cabo corresponde con la duración de cada experimento en el que se incluyen entre 315 y 525 periodos de olas aproximadamente, en una columna de agua de $3.5 \mathrm{~cm}$ en un rango de profundidad entre 2.8 y 8.6 $\mathrm{cm}$. En $43 \%$ de los experimentos se identificó el sub-rango inercial tanto en $S_{u^{\prime} u^{\prime}}\left(k_{1}\right)$ como en $S_{w^{\prime} w^{\prime}}\left(k_{1}\right)$ mientras que el carácter natural de la turbulencia, induce la ausencia intermitente del sub-rango inercial en el espectro de una o ambas componentes de la velocidades fluctuantes.

Babanin y Hauss [11], identificaron sub-rangos inerciales en el intervalo $800<k<2600 \mathrm{rad} / \mathrm{m}$, siendo 2600 $\mathrm{rad} / \mathrm{m}$ la máxima escala resuelta en los espectros. El ancho del sub-rango inercial que encontraron presentó un orden de magnitud de $10^{3} \mathrm{rad} / \mathrm{m}$. En nuestros resultados, el sub-rango inercial en $S_{u^{\prime} u^{\prime}}\left(k_{1}\right)$ y $S_{w^{\prime} w^{\prime}}\left(k_{1}\right)$ se identificó en el intervalo $753 \pm 588<k_{1}<2300 \pm 1600 \mathrm{rad} / \mathrm{m}$, con un ancho promedio de $1500 \mathrm{rad} / \mathrm{m}$. En términos generales, el sub-rango inercial en $S_{u^{\prime} u^{\prime}}\left(k_{1}\right)$ y $S_{w^{\prime} w^{\prime}}\left(k_{1}\right)$ se identificó en un intervalo similar al de los resultados de Babanin y Hauss [11], y con un ancho del mismo orden de magnitud (generalmente cuando $\xi>0.1$ ).

A partir del sub-rango inercial en sus espectros de potencia de la velocidad, Babanin y Hauss [11], estimaron valores de $\varepsilon$ con órdenes de magnitud entre $10^{-4}$ y $10^{-3} \mathrm{~m}^{2} / \mathrm{s}^{3}$. En nuestros experimentos, los valores de $\varepsilon$ obtenidos a partir del sub-rango inercial identificado en $S_{u^{\prime} u^{\prime}}\left(k_{1}\right)$ y $S_{w^{\prime} w^{\prime}}\left(k_{1}\right)$, 
presentaron órdenes de magnitud de $10^{-4} \mathrm{~m}^{2} / \mathrm{s}^{3}$, y únicamente $20 \%$ y $8 \%$ de dichos valores respectivamente, presentaron órdenes de magnitud de $10^{-3} \mathrm{~m}^{2} / \mathrm{s}^{3}$ especialmente en las menores profundidades, las que corresponden a la parte superior y más cercana a la superficie del agua. Nuestra estimación de $\varepsilon$ puede considerarse como un límite inferior con respecto a los valores calculados por Babanin y Hauss [11].

Se establece que los espectros de potencia $S_{u^{\prime} u^{\prime}}\left(k_{1}\right)$ y $S_{w^{\prime} w^{\prime}}\left(k_{1}\right)$ corresponden a un movimiento turbulento de las partículas de fluido, apreciándose sub-rangos inerciales de forma intermitente, con lo que se infiere que el movimiento inducido por olas que aún no rompen puede generar turbulencia isotrópica desarrollada independientemente del valor de la pendiente de las olas en las distintas profundidades en donde se realizaron las mediciones. Con los resultados de $S_{u^{\prime} u^{\prime}}\left(k_{1}\right)$ y $S_{w^{\prime} w^{\prime}}\left(k_{1}\right)$ se demuestra que el tamaño de los remolinos cuya energía se transfiere a remolinos de menores escalas, es mayor al aumentar la pendiente de las olas.

\section{Agradecimientos}

Este trabajo representa una contribución del proyecto RugDiSMar (CONACYT CB-2010-01-155793). Se agradece el apoyo por parte del Posgrado en Oceanografía Física del CICESE, así como el apoyo económico para estudios de Posgrado por parte del CONACYT. A la Facultad de Ciencias Marinas (FCM), al Dr. Rafael Hernández Wall y M.C. Patricia Alvarado Graef, de la Universidad Autónoma de Baja California por facilitar el desarrollo de los experimentos en el laboratorio de olas de la FCM. Se agradece especialmente a L.A. Julieta Castro por su apoyo en procedimientos de logística y administración durante las actividades propias de esta investigación. Esta investigación ha sido financiada por el proyecto 201441 SENER-CONACYT-Hidrocarburos, y el proyecto 249795 CONACYT-SENER Sustentabilidad Energética. La versión final de este trabajo se completó durante el programa Planetary Boundary Layers in Atmospheres, Oceans, and Ice on Earth and Moons (KIPT-UCSB 2018, NSF PHY1748958).
1. D. Peregrine and L. Svendsen, Proc. 16th ICCE. 1 (1978) 540550.

2. O. Reynolds, Phil. Trans. Roy. Soc. London. 174 (1883) 935982.

3. R. J. Rapp, W. K. Melville, Philos. Trans. R. Soc. Lond. A 331 (1990) 735-80.

4. L. H. Kantha, and C. A. Clayson, Ocean Modell. 6 (2004) 101124.

5. Y. Noh, H. Min, and S. Raasch, J. Phys. Oceanogr. 34 (2004) 720-735.

6. J. Gemmrich, J. Phys. Oceanogr. 40 (2010) 583-595.

7. O. M. Phillips, J. Geophys. Res. 66 (1962) 2889-2893.

8. V. V. Yefimov, and G. N. Khristoforov, Izv. Acad. Sci. USSR Atmos. Oceanic Phys., Engl. Transl. 7 (1971) 1290-1310.

9. L. Cavaleri, and S. Zecchetto, J. Geophys. Res. 92 (1987) 38943904.

10. A. V. Babanin, Geophys. Res. Lett. 33 (2006) L20605.

11. A. V. Babanin, and B. K. Haus, J. Phys. Oceanogr. 39 (2009) 2675-2679.

12. D. Dai, F. Qiao, W. Sulisz, L. Han, and A. Babanin, J. Phys. Oceanogr. 40 (2010) 2180-2188.

13. I. B. Savelyev, E. Maxeiner, and D. Chalikov, J. Geophys. Res. 117 (2012) C00J13.

14. F. Veron, F., W. Melville, and L. Lenain, J. Atmos. Oceanic Technol. 25 (2008) 307-326.

15. J. Beyá, W. Peirson, and M. Banner, Exp. Fluids. 52 (2012) 1319-1330.
16. B. Kinsman, Wind Waves: Their generation and propagation on the ocean surface. (Prentice-Hall, Englewood Cliffs, N.J. 1965).

17. Y. Goda, and Y. Suzuki, Proc. 15th ICCE, ASCE, Hawaii. (1976) 628-650.

18. E. P. D. Mansard, and E. R. Funke, Proc. 17th ICCE, ASCE, Syndey, Australia. (1980) 154-172.

19. F. Veron, and W. K. Melville, J. Atmos. Ocean. Tech. 16 (1999) 1580-1597.

20. N. Hashimoto, T. Nagai, and T. Asai, Coast. Eng. Chapter 18 (1994) 223-246.

21. C.-Y. Lin, and C.-J. Huang, Coast. Eng. 51 (2004) 395-406.

22. A. N. Kolmogorov, Dokl. Akad. Nauk SSSR. 30 (1941) 9-13.

23. A. S. Monin, and R. V. Ozmidov, Turbulence in the ocean (D. Reidel Publ. Co. Dordrecht 1985) pp. 458.

24. J. O. Hinze, Turbulence (McGraw-Hill, 1975) 790pp.

25. G. I. Taylor, Proc. 5th Int. Congress App. Mech., Cambridge Mass. (edited by J.P. Den Hartog and H. Peters, John Wiley, New York. 1983) 294-310.

26. S. R. Green, PHOENICS. J. CFD \& Appl. 5 (1992) 294-312.

27. A. Y. Benilov, and B. N. Filyushkin, Izv. Acad. Sci. USSR Atmos. Oceanic Phys., Engl. Transl. 6 (1970) 477-482.

28. E. B. Thornton, J. Geophys. Res. 84 (1979) 4931-4938.

29. J. L. Lumley, and E. A. Terray, J. Phys. Oceanogr. 13 (1983) 2000-2007. 